\title{
IDENTIFICATION OF STRUCTURAL PARAMETERS AND INPUT GROUND MOTION FROM RESPONSE TIME HISTORIES
}

\author{
By Kenzo TOKI*, Tadanobu SATO** and Junji KIYONO***
}

\begin{abstract}
We report a procedure by which structural parameters and input ground motion are identified from measured responses only. We have assumed that the coda of the response time history represents the free vibration response of the structural system. Because the coda is not effected by the input ground motion, we can first identify such structural parameters as the masses, damping coefficients and spring constants from this part of the record. Input ground motion then is estimated from the full record and the identified parameters. The identification and estimation are made with the Kalman filter. To verify the effectiveness of this procedure, we have simulated the responses of a linear, three-degree-of-freedom system for different earthquake inputs and made estimations using the simulated responses as observed records. The estimated accelerograms, the identification of which usually more difficult than the identifications of velocitigrams and displacementgrams, are in good agreement with the recorded ones for the actual earthquakes.

Keywords : identification, Kalman filter, structural parameters, input ground motion
\end{abstract}

\section{INTRODUCTION}

The relation of input motion to the output motion of a structural system is illustrated in Fig. 1. The input motion may be the ground motion induced by an earthquake, microtremor, mechanical vibration, etc., whereas the output motion is the response of a structural system due to that input. A procedure presented herein identifies, first, such structural parameters as the natural frequency and damping constant, and then estimates input motion using only the seismic response time series. Kalman filter analysis, a method that updates the estimated value at each time step by incorporating new observation data, is used in our procedure.

Many techniques have been proposed for identifying the structural parameters in the field of earthquake engineering. Toki and Sato ${ }^{11}$ developed a procedure for identifying structural parameters that uses an AR-MA process. This procedure has proved useful for detecting the dynamic parameters of structural systems; but, as the input motion is assumed to be a white noise, it can not be used when input characteristics are uncertain. Hoshiya and Saito ${ }^{2)}{ }^{3)}$ used an extended Kalman filter to solve the identification problem for a dynamic structural system. They proposed a weighted global iteration procedure to obtain fast convergency to the optimum solution and for the stability of that solution; in addition, the sensitivity of the initial condition to the convergency was studied. Hoshiya and Maruyama ${ }^{4)}$ also developed a method by which to identify the parameters of a versatile hysteretic model by use of an extended Kalman filter. In the geomechanics field, Murakami and Hasegawa ${ }^{5)}$ have proposed a new back

* Member of JSCE, Dr. Eng., Professor, Disaster Prevention Research Institute, Kyoto University, (Uji, Kyoto 611, Japan)

** Member of JSCE, Dr. Eng., Associate Professor, Disaster Prevention Research Institute, Kyoto University, (Uji, Kyoto 611, Japan)

*** Member of JSCE, M. Eng., Research Associate, Disaster Prevention Research Institute, Kyoto University, (Uji, Kyoto 611, Japan) 
analysis method which uses Kalman Filter-Finite Elements and determines the optimal location of observation nodal points. All these procedures require both input and output data. Our method differs in that the system parameters are identified from output data alone.

To the best of authors' knowledge, there have been few

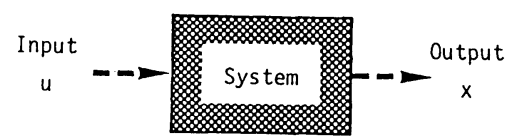

Fig. 1 Relation between input and output motion

earthquake engineering studies ${ }^{6,7)}$ whose purposes were to estimate input motion from the structural response.

This is because the input motion is known when analyzing seismic response, and earthquake engineers usually are interested in estimating the response of a structure. But it is necessary to identify the real input motion to a structure or its foundation, because the motion of the base of structure is different from the motion of free field. We have used the Kalman error filter, developed by Ott and Meder ${ }^{8)}$, to estimate input motion from the structural response and structural parameters. We have extended this method so that the input motion can be calculated from the estimation error obtained in the Kalman filter algorithm. The model used for calculation has three masses and three degrees of freedom. Its responses are assumed to be recorded in each mass.

\section{IDENTIFICATION OF STRUCTURAL PARAMETERS}

\section{(1) Identification by the Kalman filter}

When a system consists of elements, each of which is expressed by a governing equation (such as Newton's for a kinematic system), that equation is entirely described by the differential equation and the initial condition ${ }^{9}$.

Consider the signal generation and observation processes written by the linear stochastic differential equation

$$
\frac{d\{X(t)\}}{d t}=[A]\{X(t)\}+[B]\{w(t)\}
$$

and

$\{y(t)\}=[H]\{X(t)\}+\{v(t)\}$

in which vectors $\{X\},\{w\},\{y\}$ and $\{v\}$ are the stochastic process, and matrices $[A],[B]$ and $[H]$ are deterministic. By solving Eq. (1), both equations can be written as a discrete linear system;

$$
\begin{aligned}
& \left\{X_{t}\right\}=\left[\Phi_{t \mid t-1}\right]\left\{X_{t-1}\right\}+[G]\left\{W_{t-1}\right\} \\
& \left\{Y_{t}\right\}=[H]\left\{X_{t}\right\}+\left\{V_{t}\right\} \cdots \cdots \cdots \cdots \cdots
\end{aligned}
$$

in which $\left\{X_{t}\right\}$ is the $n$-dimensional state estimate vector at $t ;\left\{Y_{t}\right\}$ the $p$-dimensional observation vector at $t ;\{W\}$ and $\{V\}$ the $m$ - and $p$-dimensional Gaussian white noise with $E\left\{W_{s} W_{t}^{T}\right\}=Q \delta_{s t}$, and $E\left\{V_{s} V_{t}^{T}\right\}=R \delta_{s t}$, when $E$ represents the expectation and $\delta$ Kronecker's delta; $[\Phi]$ the state transition matrix with the dimensions $n \times n ;[H]$ the measurement matrix with the dimensions $p \times n$; and $[G]$ the system noise matrix with the dimensions $n \times m$.

If we transform the governing differential equations to Eqs. (3) and (4) by the appropriate modeling, the identification problem can be solved by use of the Kalman filter algorithm shown in Table 1.

A 3-story structural model is shown in Fig. 2. It is a system with three degrees of freedom, and we assume that the displacement or velocity response time histories are obtained at each mass. When the structure is represented as a lumped mass model which has springs and dashpots, its motion is governed by the differential equation.

$$
[M]\left\{\ddot{X}_{1}\right\}+[C]\left\{\dot{X}_{1}\right\}+[K]\left\{X_{1}\right\}=-[M]\{\ddot{u}\}
$$

in which $\left\{X_{1}\right\}$ represents the response of the structure, $\{\ddot{u}\}$ the input ground acceleration, $[M]$ the mass, $[C]$ the damping coefficient, and $[K]$ the stiffness matrix. $[M],[C]$ and $[K]$ are given by 
Table 1 Kalman filter algorithm.

(1) Store the filter state

$X(k \mid k), P(k \mid k)$

(2) Compute the predicted state

$x(k+1 \mid k)=\Phi(k+1 \mid k) X(k \mid k)$

(3) Compute the predicted error covariance matrix

$P(k+1 \mid k)=\Phi(k+1 \mid k) P(k \mid k) \Phi^{\top}(k+1 \mid k)+\Gamma(k) Q(k+1) \Gamma^{\top}(k)$

(4) Compute the Kalman gain matrix

$K(k+1)=P(k+1 \mid k) M^{\top}(k+1)\left[M(k+1) P(k+1 \mid k) M^{\top}(k+1)+R(k+1)\right]^{-1}$

(5) Process the observation $y_{k+1}$

$x(k+1 \mid k+1)=x(k+1 \mid k)+k(k+1)\left[y_{k+1}-M(k+1) x(k+1 \mid k)\right]$

(6) Compute the new error covariance matrix

$P(k+1 \mid k+1)=[1-k(k+1) M(k+1)] P(k+1 \mid k)$

(7) Set $k=k+1$, and return to step (1).

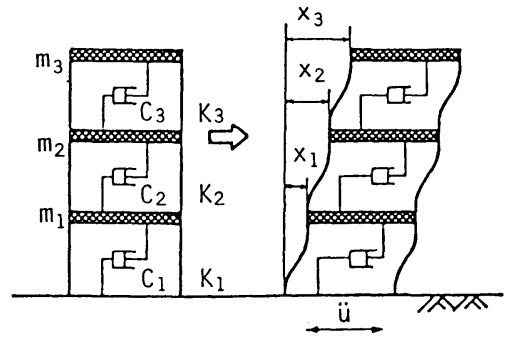

Fig. 2 Structural model.

$$
\begin{aligned}
& {[M]=\left[\begin{array}{ccc}
m_{1} & 0 & 0 \\
0 & m_{2} & 0 \\
0 & 0 & m_{3}
\end{array}\right], \quad[C]=\left[\begin{array}{ccc}
c_{1}+c_{2} & -c_{2} & 0 \\
-c_{2} & c_{2}+c_{3} & -c_{3} \\
0 & -c_{3} & c_{3}
\end{array}\right],} \\
& {[K]=\left[\begin{array}{ccc}
k_{1}+k_{2} & -k_{2} & 0 \\
-k_{2} & k_{2}+k_{3} & -k_{3} \\
0 & -k_{3} & k_{3}
\end{array}\right] \ldots \ldots \ldots \ldots \ldots \ldots \ldots \ldots \ldots \ldots \ldots \ldots \ldots \ldots \ldots \ldots \ldots \ldots}
\end{aligned}
$$

The state variables corresponding to Eqs. (3), (4) are

$$
\{X\}=\left\{\left\{X_{1}\right\}\left\{X_{2}\right\}\left\{X_{3}\right\}\left\{X_{4}\right\}\right\}^{T}
$$

in which

$$
\begin{aligned}
& \left\{X_{1}\right\}=\left\{\begin{array}{lll}
x_{1} & x_{2} & x_{3}
\end{array}\right\}^{T} \ldots \\
& \left\{X_{2}\right\}=\left\{\begin{array}{lll}
\dot{x}_{1} & \dot{x}_{2} & \dot{x}_{3}
\end{array}\right\}^{T}
\end{aligned}
$$

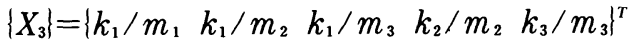

$$
\begin{aligned}
& \left\{X_{4}\right\}=\left\{\begin{array}{lll}
c_{1} / m_{1} & c_{2} / m_{2} & c_{3} / m_{3}
\end{array}\right\}^{T}
\end{aligned}
$$

The first differentiation of the state variables is written from Eqs. (5) and (7);

$$
\{\dot{X}\}=\left\{\begin{array}{l}
\left\{\dot{X}_{1}\right\} \\
\left\{\dot{X}_{2}\right\} \\
\left\{\dot{X}_{3}\right\} \\
\left\{\dot{X}_{4}\right\}
\end{array}\right\}=\left\{\begin{array}{c}
\left\{X_{2}\right\} \\
-[M]^{-1}[C]\left\{X_{2}\right\}-[M]^{-1}[K]\left\{X_{1}\right\}-\{\ddot{u}\} \\
\{\mathbf{0}\} \\
\{\mathbf{0}\}
\end{array}\right\}
$$

The structural parameters can now be identified according to the method proposed by Hoshiya and Saito ${ }^{3)}$. The parameters which must be identified are the stiffness $\left\{X_{3}\right\}$ and damping $\left\{X_{4}\right\}$ terms. The values for each mass also are unknown, but the number of variables contained in the state variable $\left\{X_{4}\right\}$ can be condensed by use of the relation ${ }^{3)}$;

$$
\frac{c_{1}}{m_{2}}=\frac{\left(c_{1} / m_{1}\right)\left(k_{1} / m_{2}\right)}{k_{1} / m_{1}}, \quad \frac{c_{1}}{m_{3}}=\frac{\left(c_{1} / m_{1}\right)\left(k_{1} / m_{3}\right)}{k_{1} / m_{1}}
$$

The continuous differential equation, Eq. (12), is rewritten as follows;

$$
\frac{d\{X\}}{d t}=\{f\}
$$

By use of the following relation, we can descretize the continuous equation and get the state transition matrix $^{10)}$.

$$
\begin{aligned}
& {\left[A_{i j}\right]=\frac{\partial f_{i}}{\partial x_{j}} \ldots \ldots \ldots \ldots \ldots \ldots \ldots \ldots \ldots \ldots \ldots} \\
& {[\Phi]=I+\Delta t \cdot[A]+\frac{\Delta t^{2}}{2 !} \cdot[A]^{2}}
\end{aligned}
$$

in which $\{f\}$ is a vector function and $\Delta t$ the time interval.

Even when ground motion stops after an earthquake, a structure continues to oscillate because of free 
vibration. Because the right side of Eq. ( 5 ) becomes zero during free vibration, the equation of motion is

$[M]\left\{\ddot{X}_{1}\right\}+[C]\left\{\dot{X}_{1}\right\}+[K]\left\{X_{1}\right\}=\{0\}$

Therefore, we can identify the system parameters by eliminating the input term incorporated in the state variable. The right side of Eq. (12) is replaced by

$$
\left\{\begin{array}{c}
\left\{X_{2}\right\} \\
-[M]^{-1}[C]\left\{X_{2}\right\}-[M]^{-1}[K]\left\{X_{1}\right\} \\
\{\mathbf{0}\} \\
\{\mathbf{0}\}
\end{array}\right\}
$$

The components of the observation matrix vary depending on the type of record selected. Displacement or velocity records may be used as observation data. We have selected displacement records, for which the observation matrix is

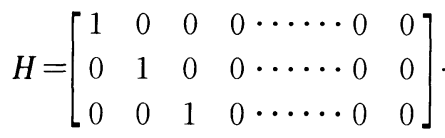

\section{( 2 ) Analysis procedure and results}

In solving for the unknown parameters included in the state variables, we adopted a weighted global iteration procedure $^{3)}$ to obtain a stable solution regardless of the initial conditions. This procedure accelerates the convergence speed by multiplying the estimated error covariance with weight and renews the initial condition with global iteration. To decide the number of global iterations we investigated the process of convergence. The model is the same as in Fig. 2 ; its structural parameters are given in Table 2. The input time history is shown in Fig. 3.

The input motion is simulated by multiplying the white noise by an evolutionary function. The spectrum of this wave has the characteristics of white noise. The response time histories for each story are shown in Fig. 4(a ), ( b ), ( c ). These waves are added with the white noise of the intensity of $1 \%$ of mean square value of the response which observed at the first story. The input motion is identified by regarding the calculated displacement responses as observed records. The process of convergence $\left\{k_{i} / m_{i}\right\}(i=1,2,3)$ was checked to confirm the stability of the solution. These parameters are identified using response time history 2 seconds from $12 \mathrm{sec}$ to $10 \mathrm{sec}$. This process, given in Fig. 5, shows that stable solution are obtained in a few global iterations. But the parameters don't converge to the exact value. This is because the convergence is affected by the beginning time of filteration, filtering period and the ratio of signal and

\begin{tabular}{|c|c|c|c|}
\hline & mass 1 & mass 2 & mass 3 \\
\hline $\begin{array}{l}\text { mass: } m i \\
\left(t \cdot \sec ^{2} / m\right)\end{array}$ & 10 & 10 & 10 \\
\hline $\begin{array}{l}\text { damping } \\
\text { coeff.: } s_{i} \\
(t \cdot s e c / m)\end{array}$ & 40 & 40 & 30 \\
\hline $\begin{array}{l}\text { spring } \\
\text { const.: } d_{i} \\
(t / m)\end{array}$ & $\begin{array}{llll}3 & 0 & 0 & 0\end{array}$ & 2000 & 1000 \\
\hline
\end{tabular}

Table 2 Parameters of the structural model.

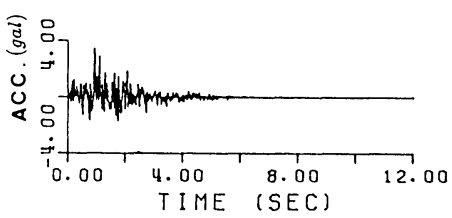

Fig. 3 Input ground acceleration.
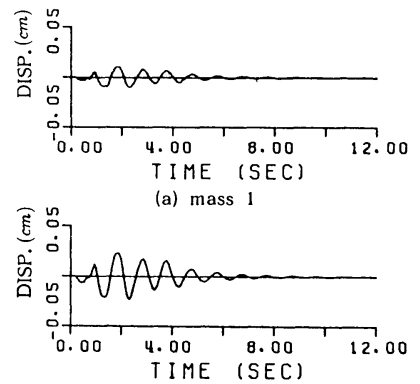

(b) mass 2

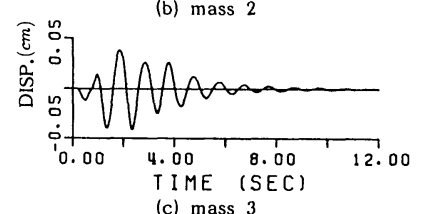

(c) mass 3

Fig. 4 Response displacement at each story of the structure 
noise level. In this case, the lower story has smaller ratio of the response and the noise. We used the weight $W=20$, the resulting number of global iteration being $4 \sim 6$. The covariance of the observation error, $R$, was chosen to be between $10^{-6}$ and $10^{-2}$.

It is difficult to determine the exact time at which the input motion becomes zero from the response time history. To surmount this, we used the free vibration part of the response time history as the input to the Kalman filter and filtered it inversely for the time axis. Fig.6(a) and (b) show the identified results obtained by filtering the record for the portion from $t_{b}$ to $t_{e} ; t_{b}$ representing the beginning and $t_{e}$ the end of filteration. Fig. 6 (a) corresponds to the parameter of stiffness $\{k / m\}$ and ( b ) to damping $\{c / m\}$. Their abscissas are $t_{e}$. The line parallel to the $t_{e}$ axis expresses the exact value. The results are not effected strongly by the choice of the beginning time, $t_{b}$. In this case, the range of time, $t_{b}$, which gives reliable values for the identified parameters is $12 \sim 6 \mathrm{sec}$. We even can identify unknown parameters from a rough estimate of $t_{b}$ which represents a response amplitude of close to zero. A filtering period of only a few seconds is sufficient.

The input time history from $12 \mathrm{sec}$ back to $0 \mathrm{sec}$ is shown at the bottoms of Fig. 6(a) and (b). In comparison with the upper three values, the deviation of the estimated from the exact value becomes large about the time when the effect of the input motion is included in the response time history.

Because noise information is needed when using the Kalman filter, an estimation of the value of noise convariance must be made in advance. The accuracy of this identification was examined by using the initial value that coincides with the exact value (Fig. 7). The parameter examined is damping, $c_{1} / m_{1}$. In this case, the results of identification do not deviate from the exact value. Filtering starts from $12 \mathrm{sec}$ and continues inversely on the time axis. The observation error covariance, $R$, representing the covariance of noise added to the observation value in Eq. (2) or Eq. (4), must be very small in the time range in which the effect of the input motion can be ignored. The identified results, however, gradually diverge from the exact value as filtering proceeds. If we make the value of $R$ larger, the identified results again coincide

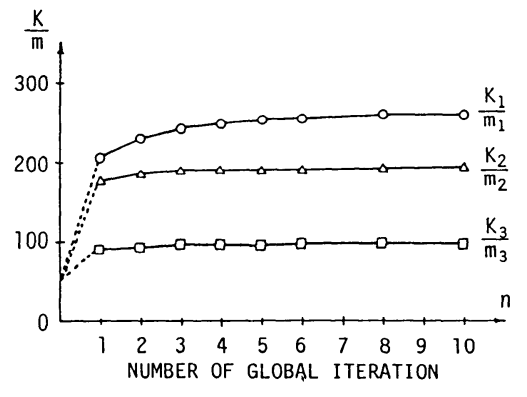

Fig. 5 Convergence process of $\{k / m\}$ for the number of global iterations.

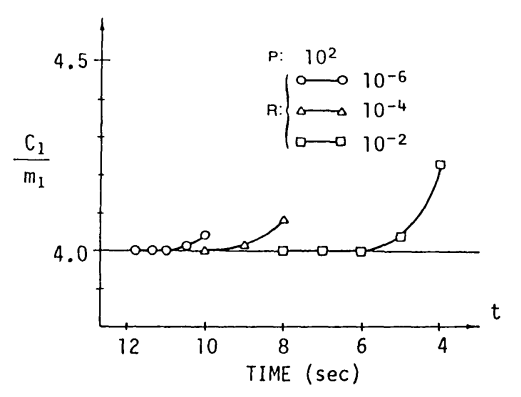

Fig. 7 Transition of identification results for various observation error covariance $R \mathrm{~s}$.
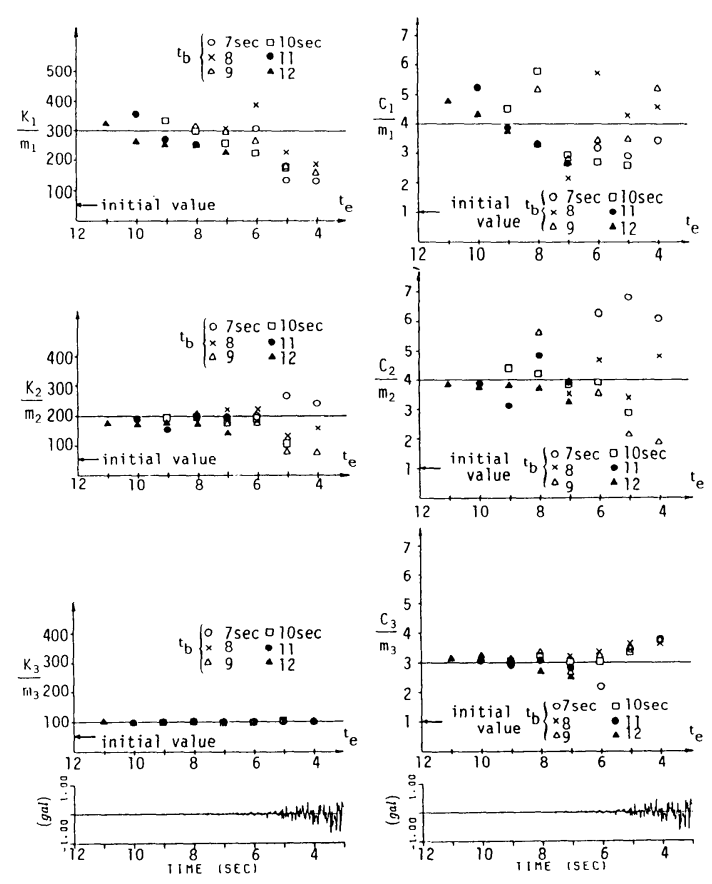

(a)stiffness $\{k / m\}$

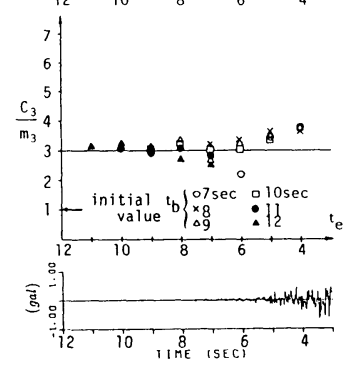

(b)damping $\{c / m\}$

Fig. 6 Relation between the identification results and $t_{b}, t_{e}$ 
with the exact value. But even when a large value is assumed for $R$, the initial value can not be maintained beyond $6 \mathrm{sec}$. This is because the input motion affects the response time history even if the input amplitude is small; but, we can bury this small amplitude in the inherent noise. When the amplitude of the input motion is so small as to be negligible, we can assign a very small value to $R$. When the inverse filtering time passes $10 \mathrm{sec}$ and the input motion can no longer be ignored, we can choose a larger value for $R$ and eliminate the effect of that motion. By making the value of $R$ large, we can keep the identified values constant only up to $6 \mathrm{sec}$ without deviation from the initial values. This explains why the identified values become unreliable from 6 sec (Fig. 6).

\section{ESTIMATION OF INPUT MOTION}

\section{(1) Equation for the error filter}

Once the structural parameters have been identified from the response alone, we can calculate the input time history by using both the identified parameters and responses ${ }^{7)}$.

Consider the following system;

$\{\dot{X}(t)\}=[A]\{X(t)\}+\{u(t)\}$

$\{y(t)\}=[H]\{X(t)\}+\{v(t)\}$

Assuming that $\{X(t)\}$ and $\{u(t)\}$ are step functions and multiplying Eq. (20) by $e^{-A t}$, we get

$\left\{X_{t}\right\}=\left[\Phi_{t \mid t-1}\right]\left\{X_{t-1}\right\}+\left\{h_{t-1}\right\}$

$\left\{Y_{t}\right\}=\left[H_{t}\right]\left\{X_{t}\right\}+\left\{V_{t}\right\}$

$\left\{h_{t}\right\}$ in Eq. (22) is given by

$$
\left\{h_{t-1}\right\}=\int_{t-\Delta t}^{t}[\Phi(t, \tau)]\{u(\tau)\} d \tau
$$

As $u(t)$ is the step function, the numerical integration of Eq. (24) becomes

$$
\left\{h_{t-1}\right\}=[\Phi]\{u\} \Delta t
$$

$\mathrm{Eq}$. (22) is rewritten

$$
\left\{h_{t-1}\right\}=\left\{X_{t}\right\}-\left[\Phi_{t \mid t-1}\right]\left\{X_{t-1}\right\}
$$

Because the exact value of $\left\{X_{t}\right\}$ is unknown, we replace $\left\{X_{t}\right\}$ and $\left[\Phi_{t \mid t-1}\right]\left\{X_{t-1}\right\}$ with $\{X(t \mid t)\}$ and $\{X(t \mid t-1)\}$ (see Table 1). Eq. (26) is rewritten

$$
\left\{h_{t-1}\right\}=\{X(t \mid t)\}-\{X(t \mid t-1)\}
$$

We can obtain the input motion $u(t)$ by substituting Eq. (27) into Eq. (25).

$$
\left.\{u\}=\frac{1}{\Delta t}[\Phi]^{-1}\{\mid X(t \mid t)\}-\{X(t \mid t-1)\}\right\}
$$

\section{(2) Method of analysis}

The equation of motion for the model shown in Fig. 2 is represented by Eq. ( 5 ). The matrices [M], [C], and $[K]$ are given by Eq. ( 6 ). Transforming the upper two rows of Eq. (12), the state equation becomes

$$
\left\{\begin{array}{l}
\left\{\dot{X}_{1}\right\} \\
\left\{\dot{X}_{2}\right\}
\end{array}\right\}=\left[\begin{array}{cc}
0 & I \\
-[M]^{-1}[K] & -[M]^{-1}[C]
\end{array}\right]\left\{\begin{array}{l}
\left\{X_{1}\right\} \\
\left\{X_{2}\right\}
\end{array}\right\}+\left\{\begin{array}{c}
\{0\} \\
-\{\ddot{u}\}
\end{array}\right\} \text {. }
$$

In section two, the input term, $-\{\ddot{u}\}$, was incorporated in the state transition matrix with the unknown parameter. Here the independent term has been set up as the input term. The observation equation is given by

$$
\{y\}=[B]\left\{\begin{array}{l}
\left\{X_{1}\right\} \\
\left.X_{2}\right\}
\end{array}\right\}+\{V\}
$$

If the equation of motion is expressed as Eq. (29) and calculations are done with the Kalman filter algorithm shown in Table 1, the input motion is obtained from Eqs. (28).

(3) Analytical results

The structural parameters used to estimate the input motion are shown in Table 3, the exact values being 
Table 3 Structural parameters used to estimate the input motion.

\begin{tabular}{|c|c|c|c|c|c|c|c|c|}
\hline & $\frac{K_{1}}{m_{1}}$ & $\frac{K_{1}}{m_{2}}$ & $\frac{K_{1}}{m_{3}}$ & $\frac{K_{2}}{m_{2}}$ & $\frac{K_{3}}{m_{3}}$ & $\frac{C_{1}}{m_{1}}$ & $\frac{\mathrm{C}_{2}}{m_{2}}$ & $\frac{\mathrm{C}_{3}}{m_{3}}$ \\
\hline exact value & 300. & 300. & 300. & 200. & 100. & 4.00 & 4.00 & 3.00 \\
\hline $\begin{array}{c}\text { case 1 } \\
(12 \sim 10 \text { sec })\end{array}$ & 257. & 287. & 339. & 174. & 99. & 3.75 & 3.78 & 3.11 \\
\hline $\begin{array}{c}\text { case 2 } \\
(10 \sim 8 s e c)\end{array}$ & 306. & 284. & 298. & 191. & 98. & 5.82 & 4.18 & 3.40 \\
\hline
\end{tabular}

Table 4 Identified structural parameters.

\begin{tabular}{|c|c|c|c|c|c|c|c|c|}
\hline & $\frac{\mathrm{K}_{1}}{\mathrm{~m}_{1}}$ & $\frac{\mathrm{K}_{1}}{\mathrm{~m} 2}$ & $\frac{\mathrm{K}_{1}}{\mathrm{~m} 3}$ & $\frac{\mathrm{K}_{2}}{\mathrm{~m}_{2}}$ & $\frac{\mathrm{K}_{3}}{\mathrm{~m}_{3}}$ & $\frac{\mathrm{C}_{1}}{\mathrm{~m}_{1}}$ & $\frac{\mathrm{C}_{2}}{\mathrm{~m} 2}$ & $\frac{\mathrm{C}_{3}}{\mathrm{~m} 3}$ \\
\hline $\begin{array}{c}\text { E1 Centro } \\
(60 \sim 55 \mathrm{sec})\end{array}$ & 270 & 311. & 308 & 200. & 99 & 3.97 & 3.99 & 3.03 \\
\hline $\begin{array}{c}\text { Taft } \\
(60 \sim 55 \mathrm{sec})\end{array}$ & 264. & 305. & 307. & 199. & 100. & 3.55 & 4.01 & 3.01 \\
\hline
\end{tabular}

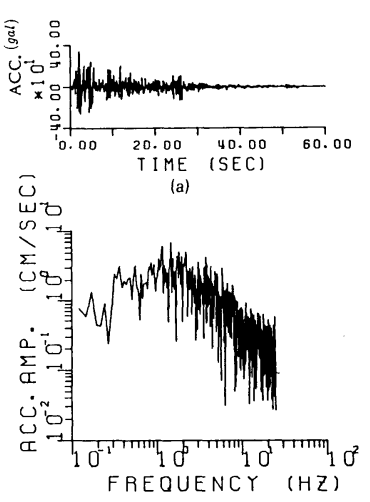

(b)

Fig. 9 Input accelerogram and its Fourier spectrum (El Centro).

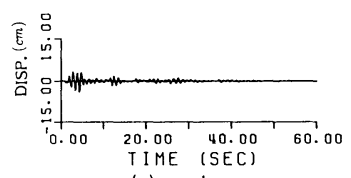

(a) mass 1

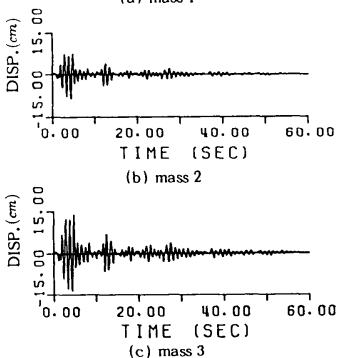

(c) mass 3

Fig. 10 Response displacement at each mass (El Centro).
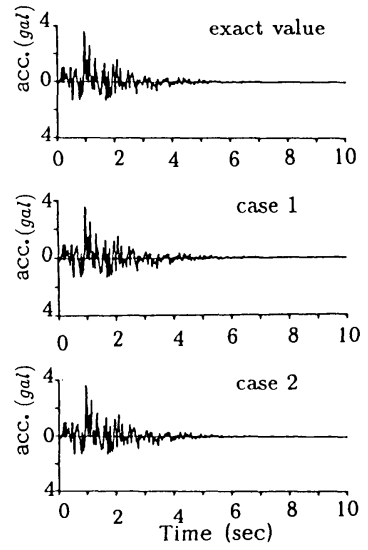

Fig. 8 Estimated input motion.
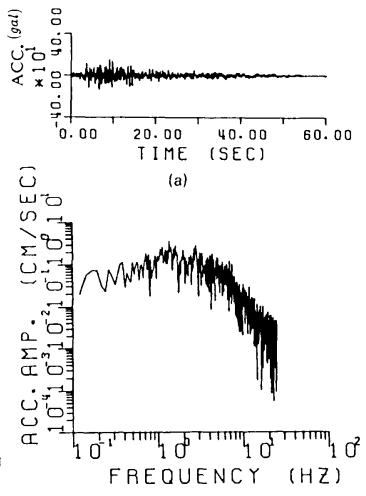

(b)

Fig. 11 Input accelerogram Fig. 12 Response displacement and its Fourier spectrum (Taft).
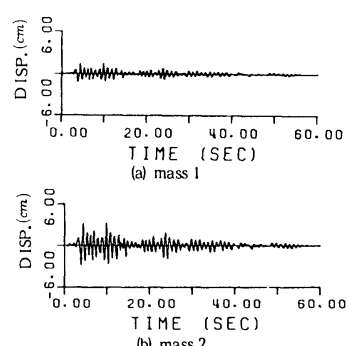
(b) mass 2

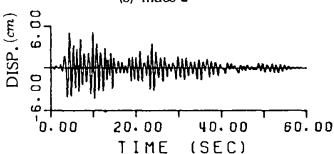

I ME (SEC) at each mass (Taft).

given in the first row. The parameters in cases 1 and 2 are the identified results obtained by use of the response time history shown in Fig. 4. Case 1 corresponds to results obtained by filtering the record from 12 to $10 \mathrm{sec}$, and case 2 from 10 to $8 \mathrm{sec}$. The maximum error for the identified parameters is about $10 \%$.

The input motions estimated with these parameters are shown in Fig. 8, the similarity of the waveforms is such that individual waves can not be distinguished at a glance. A comparison of the times corresponding to peak acceleration, the shapes of the waveforms, and the duration of each shows that any differences are negligible.

The El Centro (1940) and Taft (1952) records were used as input accelerations in the model shown in Fig. 2. We identified the structural parameters and estimated the input motion from the obtained responses alone. The structural parameters identified are shown in Table 4 ; the input motion was estimated with these parameters. The input accelerogram of the El Centro record and it's Fourier spectrum are shown in Fig. 9(a), ( b ). The response at each mass is shown in Fig. 10(a), ( b ), (c). The accelerogram and Fourier spectrum of the Taft record correspond to Fig. 11 (a), (b) and the responses to Fig. 12(a), (b), (c).

The estimated input motions and Fourier spectra are shown in Figs. 13 and 14. Structural parameters were identified using a portion of response time history for 5 seconds; from 60 to $55 \mathrm{sec}$. A comparison with the original time histories shows that the results are in good agreement for the evolution of the 
Table 5 Peak accelerations and the times corresponding to the original and estimated waves.

\begin{tabular}{|c|c|c|c|}
\hline \multicolumn{2}{|c|}{} & $\begin{array}{c}\text { Recorded } \\
\text { Motion }\end{array}$ & $\begin{array}{c}\text { Estimated } \\
\text { Accelerograa }\end{array}$ \\
\hline \multirow{2}{*}{ E1 Centro } & $\begin{array}{c}\text { Peak Acc. } \\
\text { (ga1) }\end{array}$ & 341.7 & 328.1 \\
\cline { 2 - 4 } & $\begin{array}{c}\text { Time of } \\
\text { Occurrence } \\
\text { (sec) }\end{array}$ & 2.41 & 2.41 \\
\hline \multirow{2}{*}{$\begin{array}{c}\text { Peak Acc. } \\
\text { (ga1) }\end{array}$} & $\begin{array}{c}\text { Time of } \\
\text { Occurrence } \\
\text { (sec) }\end{array}$ & 9.12 & 145.3 \\
\cline { 2 - 4 } & \multicolumn{2}{|c|}{} & 9.12 \\
\hline
\end{tabular}

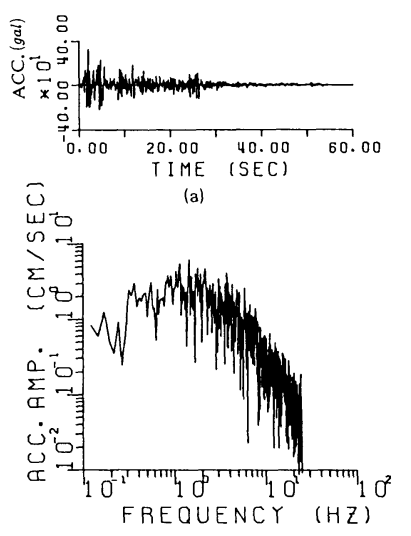

(b)

Fig. 13 Estimated input motion and $F$ its Fourier spectrum (El Centro).

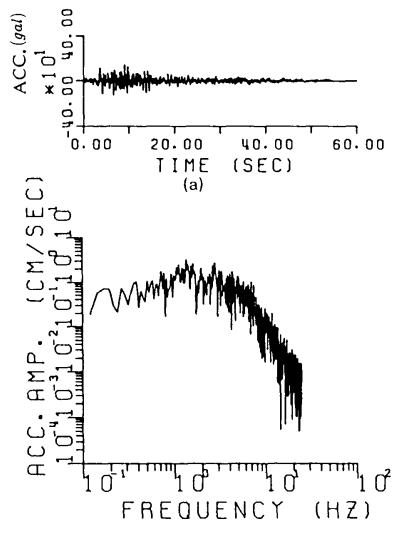

(b)

Fig. 14 Estimated input motion and its Fourier spectrum (Taft).

waveform, the time corresponding to the peak acceleration, and the duration. But, beyond $10 \mathrm{~Hz}$, high frequency spectral components show some discrepancy. The explanation is that the noise contained in the response signal is assumed to be Gaussian white noise and its spectral level is flat. The Kalman filter eliminates noise from the contaminated signal in the time domain. If we reduce the white noise component in the spectrum logarithmically, the relatively small amplitude component (beyond $10 \mathrm{~Hz}$ in Figs. 13 and 14) must decrease markedly.

The peak acceleration values and corresponding times for the original and estimated waves are given in Table 5 for the El Centro and Taft records. The differences in peak acceleration are $4 \%$ (El Centro) and $5 \%$ (Taft) of the values for the original waves; unremarkable differences. Although the peak acceleration is somewhat underestimated, the corresponding times for acceleration coincide. The decrease in the high frequency component therefore has very little effect on the identification of the input motion.

\section{CONCLUSIONS}

Our results can be summarized as follows:

(1) Structural parameters can be identified merely from the coda part of the response time history without information on the input motion. Truncation of a portion of the coda has a negligible effect on the results.

(2) The free vibration part of the response is filtered after the input motion becomes zero. But, when the amplitude of the input motion is relatively small, the effect of this motion can be eliminated by chosing the error convariance, $R$, larger.

(3) We extended the use of the Kalman error filter for the one-degree-of-freedom system proposed by Ott and Meder to a multi-degree-of-freedom system and investigated the efficiency of the latter procedure for estimating the input motion from the structural response and the identified structural parameters. In our procedure, even when the identified structural parameters deviate by about $10 \%$, the effect on the estimated input motion is negligible.

(4) The input accelerogram obtained from the error filter shows a decrease in amplitude for frequency beyond $10 \mathrm{~Hz}$. But even for the accelerogram, for which identification usually is more difficult than that of velocity and displacement, the difference in peak values is only about $4 \sim 5 \%$.

(5) The serial procedure described makes it possible both to identify structural parameters and to estimate the input motion from the seismic response of a structure alone. 


\section{REFERENCES}

1) Toki, K., Sato, T. and Oiki, Y. : Detection of Dynamic Properties of Structural Systems by the Autoregressive Moving Average Method, Annuals of Disaster Prevention Research Institute, Kyoto University, No. 21 B-2, pp. 57-68, 1987 (in Japanese).

2) Hoshiya, M. and Saito, E. : Identification Problem of Some Seismic Systems by Extended Kalman Filter, Proc. of the Japan Society of Civil Engineers, No. 339, pp.59-67, 1983 (in Japanese).

3) Hoshiya, M. and Saito, E. : Estimation of Dynamic Properties of a Multiple Degree of Freedom Linear System, Proc. of the Japan Society of Civil Engineers, No. 334/ I-1, pp. 289-298, 1984 (in Japanese).

4) Hoshiya, M. and Maruyama, O. : Identification of Restoring Force Characteristics of Nonlinear Systems during Earthquakes, Proc. of the Japan Society of Civil Engineers, No. 386/ I-8, pp.397-405, 1987 (in Japanese).

5) Murakami, A. and Hasegawa, T. : Back Analysis by Kalman Filter-Finite Elements and a Determination of Optimal Observed Points Location, Proc. of the Japan Society of Civil Engineers, No. 388/ III-8, pp. 227-235, 1987 (in Japanese).

6) Toki, K., Sato, T., Kiyono, J. and Ichihara, K. : Identification of the Fault Rupture Process by use of the Kalman Filter, Annuals of Disaster Prevention Research Institute, Kyoto University, No. 29 B-2, pp. 1-16, 1986 (in Japanese).

7) Toki, K., Sato, T. and Kiyono, J. : Identification of the Input Ground Motion of a Multi-Input System by use of the Kalman Filter, Annuals of Disaster Prevention Research Institute, Kyoto University, No. 33 B-2, 1989 (in press).

8) Ott, N. and Meder, H. G. : The Kalman filter as a Prediction Error Filter, Geophysical Prospecting 20, pp. 549-560, 1970.

9) Sagara, S., Akizuki, K., Nakamizo, T. and Katayama, T. : System Identification, Japan Society of Automatic Control, pp. 16-20, 1981.

10) Jazwinski, A. H. : Stochastic Process and Filtering Theory, Academic Press, pp. 272-281, 1970.

(Received April 27 1989) 